\title{
A Comparison of Low-Energy As Ion Implantation and Impurity-Free Disordering Induced Defects in N-Type GaAs Epitaxial Layers
}

\author{
Prakash N. K. DeEnapanraY*, B. G. SVensson ${ }^{2,3}$, H. H. TAN ${ }^{1}$ and C. JAGAdish ${ }^{1}$ \\ ${ }^{1}$ Department of Electronic Materials Engineering, Research School of Physical Sciences and Engineering, \\ The Australian National University, Canberra, A.C.T. 0200, Australia \\ ${ }^{2}$ Physics Department/Physical Electronics, P.B. 1048 Blindern, N-0316 Oslo, Norway \\ ${ }^{3}$ Royal Institute of Technology, Solid State Electronics, P.O. Box E229, SE-164 40, Kista-Stockholm, Sweden
}

(Received May 7, 2002; accepted for publication September 11, 2002)

We have compared the electrical properties of n-type GaAs layers disordered by either $40 \mathrm{keV}$ As ion implantation or an impurity-free process employing an $\mathrm{SiO}_{2}$ capping layer. Current-voltage and capacitance-voltage measurements on Au Schottky barrier diodes fabricated on the processed layers showed that the impurity-free method retained the much better electrical quality of the GaAs epitaxial layers. Different sets of defects were observed in the implanted samples and impurityfree disordered samples, which meant that the charge transfer across the Schottky barriers was different in the two cases. Our results further reveal that the concentrations and diffusion lengths of defects created by ion implantation were much larger. The impurity-free method retains the better electrical quality of the semiconductor material.

[DOI: 10.1143/JJAP.42.1158]

KEYWORDS: impurity-free disordering, defects, GaAs, Schottky barrier, diffusion

\section{Introduction}

Quantum well intermixing (QWI) has emerged as a versatile technique for the monolithic integration of optoelectronic devices and circuits. ${ }^{1)}$ It involves the band gap engineering of quantum-confined heterostructures by interdiffusing the constituent atoms at the quantum well-barrier interfaces. The band gap of the intermixed quantum well (QW) is usually larger than that of the original structure, hence providing the possibility to form low-loss optical waveguides, band gap-engineered modulators, lasers and detectors using only one epitaxial growth. ${ }^{1,2)}$ In the GaAsbased system, intermixing proceeds by the diffusion of point defects, especially the vacancies, on the group III sublattice. $^{3-5)}$ Hence, the band gap modification of III-V heterostructures is effected by defect engineering.

The defects required for interdiffusion are generally created by ion implantation or an impurity-free method which employs $\mathrm{SiO}_{2}$ capping layers. ${ }^{1,2)}$ Ion implantation offers very high reproducibility and is spatially selective. In contrast, impurity-free interdiffusion has been plagued with problems of reproducibility and spatial selectivity. ${ }^{6)}$ Some of the outstanding issues related to the reproducibility ${ }^{7-11)}$ and spatial selectivity $^{12,13)}$ of impurity-free interdiffusion have recently been addressed, and new results have helped in our better understanding of the mechanisms driving the impurity-free process. Apart from criteria related to reproducibility and spatial selectivity, ion-implantation-induced and impurity-free intermixing have recently been compared for their ability to improve the quantum efficiency and frequency response of InGaAs/GaAs ${ }^{14)}$ or $\mathrm{InGaAs}_{\mathrm{InP}}{ }^{15)}$ quantum well structures. It has been shown that the carrier capture into an intermixed quantum well was much faster than capture into the as-grown structure. The reason for this enhanced carrier capture rate was attributed to the shape of the interdiffused quantum well. In addition to the band gap engineering, intermixing also improves other optical properties, namely the quantum efficiency and frequency response, of optoelectronic devices. ${ }^{14)}$ Furthermore, the carrier collection

*E-mail address: pnk109@rsphysse.anu.edu.au efficiency of a quantum well is more efficient in samples intermixed by ion implantation compared to the impurityfree process. ${ }^{15)}$ It is also well-known that defects introduce deep levels within the band gap of semiconductor materials. These deep levels may act as efficient recombination centers for free carriers and increase the leakage current of devices. Moreover, deep levels may trap free carriers and cause free carrier compensation. ${ }^{16)}$ It is, therefore, important from the device performance point of view to, not only study the optical properties of III-V semiconductors, but also assess the influence of defects on their electrical properties.

In the present study, defects have been created in n-type GaAs epilayers by either implantation with $40 \mathrm{keV}$ As ions or the impurity-free process using $\mathrm{SiO}_{2}$ capping followed by annealing. Two different sets of defects are introduced by the two methods. We found that the free carrier compensation and leakage current was more severe for ion implantation. The results reported here demonstrate that although ion implantation may provide improved optical properties over the impurity-free method, ${ }^{14,15)}$ it does not retain the electrical quality of the semiconductor material. It is worth noting here that since disordering is performed using either ion implantation or the impurity-free method, ${ }^{1-15)}$ this study does not focus on the electrical properties of impurity-free disordered n-type GaAs previously implanted with lowenergy As ions.

\section{Experimental Details}

Epitaxial GaAs layers of (100) orientation and doped with $1 \times 10^{16} \mathrm{Si} / \mathrm{cm}^{3}$ were used in this study. The $4 \mu \mathrm{m}$ thick epitaxial layers were grown by metalorganic chemical vapour deposition (MOCVD) on $\mathrm{n}^{+}-\mathrm{GaAs}$ substrates. The epitaxial side of selected samples were capped with $200 \mathrm{~nm}$ $\mathrm{SiO}_{2}$ by plasma-enhanced chemical vapor deposition. Other samples were implanted at room temperature with $40 \mathrm{keV}$ As ions to a fluence of $5 \times 10^{13} \mathrm{~cm}^{-2}$. The samples were tilted $7^{\circ}$ off-axis in order to minimise channeling effects. The choice of ion energy, species, and dose follows from a previous study of GaAs/AlGaAs QWI. ${ }^{17)}$ Rapid thermal annealing (RTA) was performed on both $\mathrm{SiO}_{2}$-capped and 
As-implanted $\mathrm{GaAs}$ samples at $900^{\circ} \mathrm{C}$ for $30 \mathrm{~s}$ under $\mathrm{Ar}$ flow. GaAs proximity capping was used during RTA to prevent the excessive loss of As. After RTA the $\mathrm{SiO}_{2}$ layer was removed by immersing the samples in a $10 \%$ hydrofluoric acid solution. All samples were chemically cleaned, including a final dip in dilute hydrochloric acid to remove any native oxide, prior to metallization. Schottky barrier diodes (SBD) were fabricated on the epitaxial layers by thermal evaporation of $\sim 120 \mathrm{~nm}$ Au. Current-voltage $(I-V)$ and capacitance-voltage $(C-V)$ measurements were performed on the SBDs, while deep level transient spectroscopy (DLTS) measurements were made using a lock-in-type setup described in ref. 18 .

\section{Results and Discussion}

The two methods studied here create defects in a region confined close to the surface of the samples. Low-energy As ions were used in ref. 17 in order to create defects in a region spatially resolved from GaAs quantum well structures located $\sim 300 \mathrm{~nm}$ below the surface. TRIM simulations show that the projected range and straggling of $40 \mathrm{keV}$ As ions in GaAs are $\sim 20 \mathrm{~nm}$ and $\sim 11 \mathrm{~nm}$, respectively. ${ }^{19)}$ The fluence of $5 \times 10^{13} \mathrm{~cm}^{-2}$ has been shown to produce significant intermixing in GaAs/AlGaAs QWs. ${ }^{17)}$ For the impurity-free method, defects are generated close to the GaAs surface due to either the out-diffusion of $\mathrm{Ga}$ atoms into the $\mathrm{SiO}_{2}$ layer or during metallurgical reactions between the semiconductor and the encapsulant. ${ }^{1,2,5,7-11)}$ Although the exact defect creation mechanism remains unknown, there is the ample experimental evidence suggesting that the gallium vacancy, $\mathrm{V}_{\mathrm{Ga}}$, is the main defect created in GaAs by the impurity-free method. ${ }^{1,3-5)}$ It is pointed out here that the generation of defects and their diffusion in the impurity-free method occur simultaneously during the one annealing step. On the other hand, displacement damage is created by nuclear energy deposition in elastic recoils during ion implantation, and defects are "driven-in" during the post-implantation annealing step. It will be shown later that defect diffusion already takes place during ion implantation, albeit to a much lower extent than during the RTA step.

For a Schottky barrier diode, the $I-V$ technique measures the transport of free carriers over or across the barrier between the semiconductor and the metal, and is, therefore, very sensitive to the near-surface modification of the semiconductor. Figure 1 illustrates the room temperature $I-V$ characteristics of Au SBDs fabricated on the as-grown (open triangles), $\mathrm{SiO}_{2}$-capped and annealed (open diamonds), as-implanted (solid squares), and implanted and annealed (open circles) GaAs epilayers. The diode fabricated on the as-grown sample exhibits very good reverse characteristics with a current $\sim 3.5 \times 10^{-10} \mathrm{~A}$ at $-1 \mathrm{~V}$. Its linear forward characteristic curve for $V>3 k T / q$ shows that the current transport across the barrier was according to the thermionic emission theory (typical of the near-ideal SBD). The ideality factor $(n)$ and barrier height $\left(\phi_{\mathrm{b}}\right)$ of a SBD can be extracted according to the thermionic emission theory by a least squares fit to the forward characteristic curve for $V>3 k T / q^{20)}$ A summary of the electrical properties of SBDs fabricated on as-grown and processed n-type GaAs samples is given in Table I. The diode fabricated on the sample processed by the impurity-free method has degraded

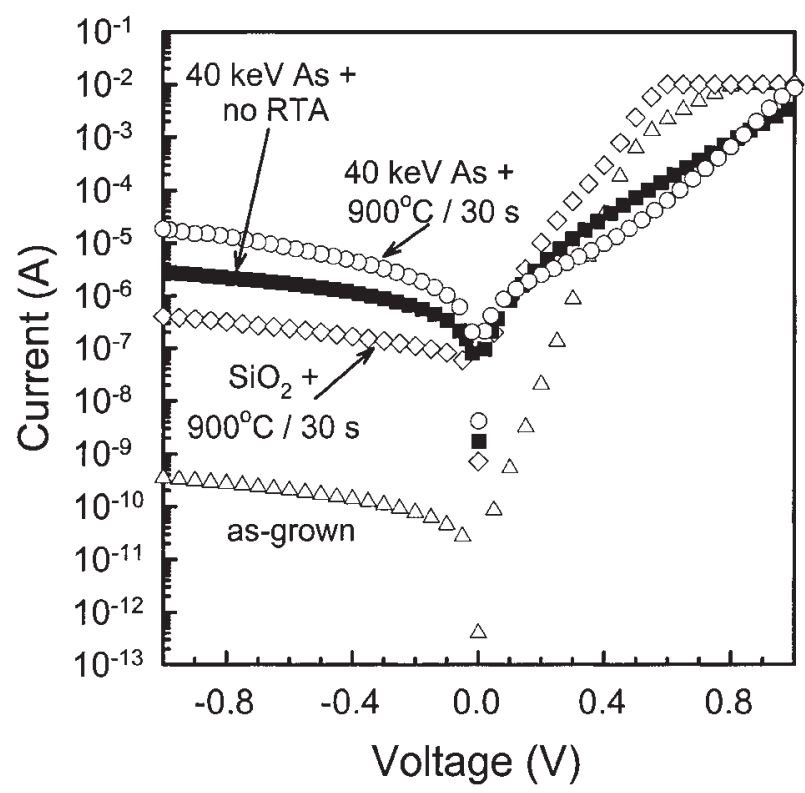

Fig. 1. Current-voltage characteristics of Schottky barrier diodes fabricated on as-grown (open triangles), $\mathrm{SiO}_{2}$-capped and annealed (open diamonds), As-implanted (solid squares), and As-implanted and annealed (open circles) n-type GaAs epitaxial layers. The ideality factor $(n)$ and barrier height $\left(\phi_{\mathrm{b}}\right)$ of the SBDs were extracted using a least squares fit to the forward characteristic curve (as-grown sample) for $V>3 k T / q$ according to the thermionic emission theory.

Table I. Summary of the electrical characteristics of SBDs fabricated on as-grown and processed n-type GaAs.

\begin{tabular}{cccc}
\hline Sample & $I_{\mathrm{R}}(-1 \mathrm{~V})(\mathrm{A})$ & $\phi_{\mathrm{b}}(\mathrm{eV})$ & $n$ \\
\hline as-grown & $3.47 \times 10^{-10}$ & 0.93 & 1.03 \\
$\mathrm{SiO}_{2}+\mathrm{RTA}$ & $4.02 \times 10^{-7}$ & 0.73 & 1.65 \\
As-implanted & $2.73 \times 10^{-6}$ & 0.64 & $>2$ \\
As-implanted+RTA & $1.85 \times 10^{-5}$ & - & -
\end{tabular}

reverse and forward characteristic curves, as indicated by the lower barrier $\left(\phi_{\mathrm{b}}=0.73 \mathrm{eV}\right)$ height and larger ideality factor $(n=1.65)$ This non-ideal behavior results from the recombination of electrons in the depletion region via deep levels. ${ }^{20)}$ The decreased slope of the forward characteristic curve above $\sim 0.2 \mathrm{~V}$ is also typical of the increase in series resistance, $R_{\mathrm{S}}$, of the impurity-free damaged GaAs layer. The $I-V$ characteristics of SBDs fabricated on the As implanted samples were even poorer, with the degradation being more significant following RTA. The barrier height of the SBDs fabricated on the As-implanted sample is $0.64 \mathrm{eV}$, and the non-ideal characteristic of diodes is reflected by their large ideality factor and reverse current. The SBDs fabricated on the As-implanted and annealed n-GaAs were the most leaking with forward $I-V$ curves exhibiting non-linearity over the entire forward voltage range (Fig. 1). Consequently, we have not been able to perform linear fittings to the forward characteristic of data points shown in open circles to extract values for $n$ and $\phi_{\mathrm{b}}$ (As-implanted + RTA) to any reasonable degree. It is worth noting here that the main aim of the present study requires the direct comparison of electrical properties of diodes fabricated on the impurity-free disordered layer, and the implanted and annealed sample. This comparison clearly shows that the implantation-induced 
disordering produced higher trap densities in n-type GaAs than impurity-free disordering, which results in the higher electron/hole pair recombination within the depletion regions of SBDs in the former case. A quantitative measure of this is the more than 200 fold increase in the reverse current of diodes fabricated on the implantation-disordered sample compared to the impurity-free disordered one.

It is also well known that defects which introduce deep levels close to the middle of the bandgap may act as efficient recombination centers. ${ }^{21)}$ Hence, the extent of free carrier compensation, $\Delta N_{\mathrm{D}}$, gives a direct indication of the concentration of electron trapping sites and their diffusion into the samples. Figure 2 illustrates the doping profiles of the same four samples as in Fig. 1. The doping profiles were extracted at room temperature from high frequency $(1 \mathrm{MHz})$ capacitance-voltage measurements. The free carrier concentration of the impurity-free disordered GaAs sample is similar to that of the as-grown samples. This result shows that the impurity-free method retained the good crystalline quality of the GaAs epilayer, i.e. defects in impurity-free disordered $\mathrm{n}-\mathrm{GaAs}$ are either created in low concentrations and/or the defects are inefficient trapping centres at room temperature. We have extracted the barrier height, $\phi_{\mathrm{b}}^{\mathrm{CV}}$, of diodes fabricated on the as-grown and impurity-free disordered GaAs layers from plots of $1 / C^{2}$ versus $V$ as described in ref. 22. The values were $\phi_{\mathrm{b}}^{\mathrm{CV}} \approx(0.90 \pm$ $0.02) \mathrm{eV}$ and $(0.74 \pm 0.02) \mathrm{eV}$ for SBDs fabricated on the as-grown and impurity-free disordered GaAs layers, respectively. These values are in good agreement with the corresponding values extracted from the $I-V$ curves in Fig. 1. Our results are consistent with the previous proposition that surface processing results in the lowering of the barrier height of diodes fabricated on n-type semiconductors. $^{23,24)}$ This reduction in barrier height has been proposed to be due to the presence of donor-type defects in the nearsurface region of the processed semiconductor. ${ }^{25)}$ The lowering of barrier height of the diode fabricated on $\mathrm{SiO}_{2}-$

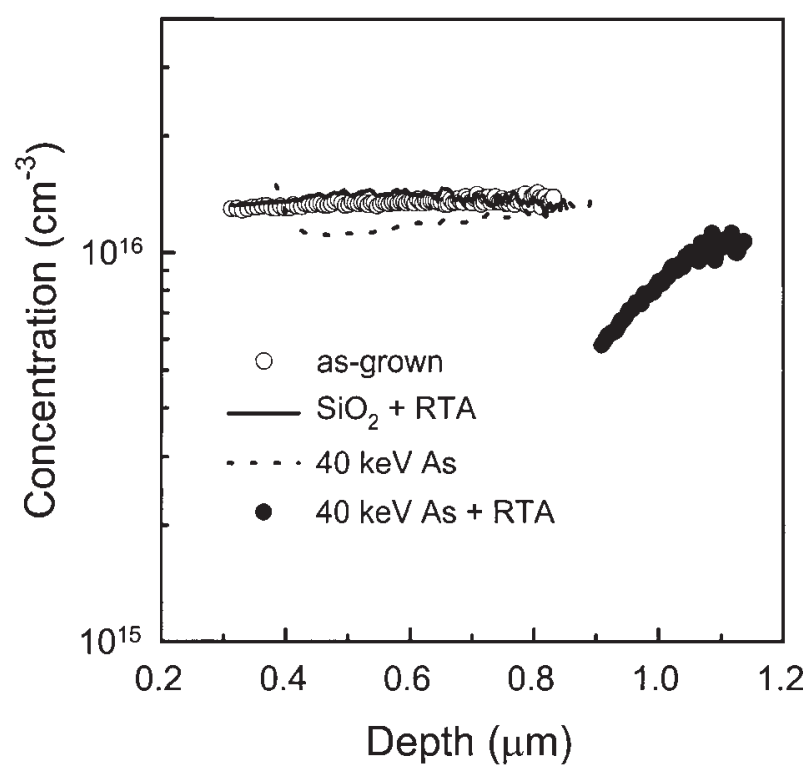

Fig. 2. Doping profiles extracted from capacitance-voltage measurements for the four samples described in legends. The high frequency $(1 \mathrm{MHz})$ measurements were performed at room temperature. capped and annealed GaAs, therefore, explains its poorer rectifying behavior (see Fig. 1). By comparison, $\Delta N_{\mathrm{D}}$ is larger for the As-implanted GaAs than the impurity-free disordered GaAs, which could account for the increased $R_{\mathrm{S}}$ seen in the forward $I-V$ curves of SBDs fabricated after ion implantation. The sample implanted with $40 \mathrm{keV}$ As (broken curve in Fig. 2) was compensated up to depths exceeding $\sim 0.8 \mu \mathrm{m}$. This shows that defects migrated to depths well beyond the projected range of As ions in GaAs during ion implantation. The apparent increase in the doping concentration towards the surface arises from the presence of deep acceptors that affect the capacitance signal within the depletion region separated from the front edge of the space-charge region. ${ }^{26)}$ After RTA defects created mostly within the top $\sim 20 \mathrm{~nm}$ of GaAs diffused beyond $1.1 \mu \mathrm{m}$. The most pronounced $\Delta N_{\mathrm{D}}$ for implanted and annealed GaAs reveals that the sample contained a larger concentration of electron trapping sites than the as-implanted sample. Consequently, diodes fabricated on the implanted and annealed sample had the poorest rectifying properties. We have not been able to extract the barrier height of diodes fabricated on the implantation-disordered sample from $C-V$ measurements because of their non-linear $1 / C^{2}$ versus $V$ curves close to zero bias. The results shown in Figs. 1 and 2 are, therefore, in good agreement.

The results shown in Figs. 1 and 2 (especially the extent of free carrier compensation) indicate that ion implantation and the impurity-free method may introduce different sets of defects with different electronic properties and concentrations. Figure 3 illustrates DLTS spectra of defects created by the impurity-free method [curve (b)] and by ion implantation both before [curve (c)] and after [curve (d)] RTA. Spectrum (a) was taken from the as-grown GaAs epilayers and shows the presence of only the EL2 with a concentration $\sim 2 \times$

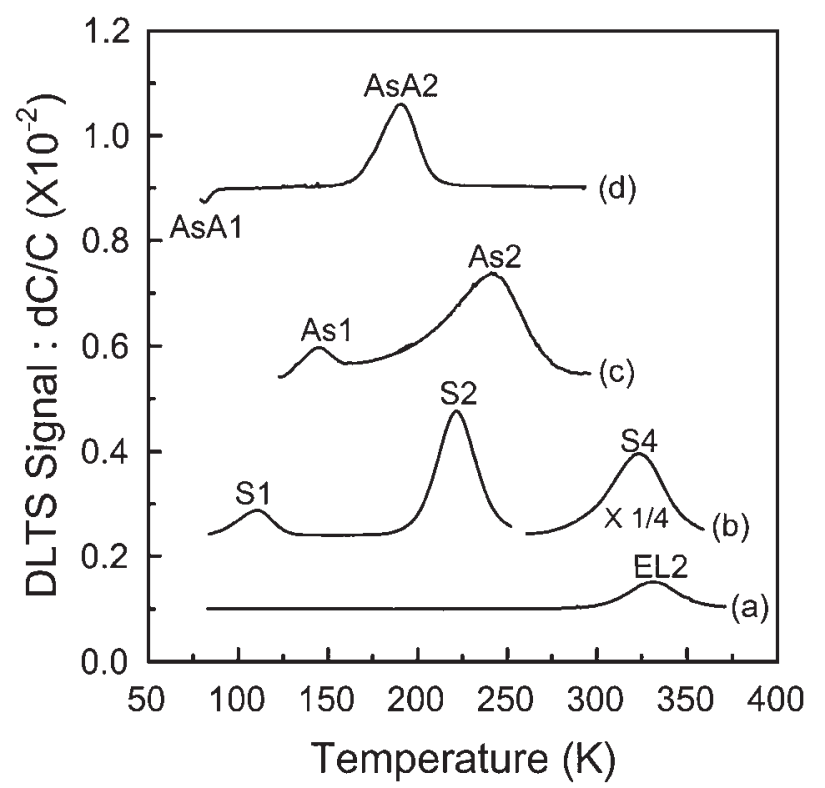

Fig. 3. DLTS spectra taken from the as-grown [curve (a)], $\mathrm{SiO}_{2}$-capped and annealed [curve (b)], As-implanted [curve (c)], and As-implanted and annealed [curve (d)] samples. The spectra correspond to the $(3.2 \mathrm{~s})^{-1}$ rate window and have been offset intentionally for clarity. DLTS measurements could not be performed above room temperature for the ion implanted samples because of their leaky character. 


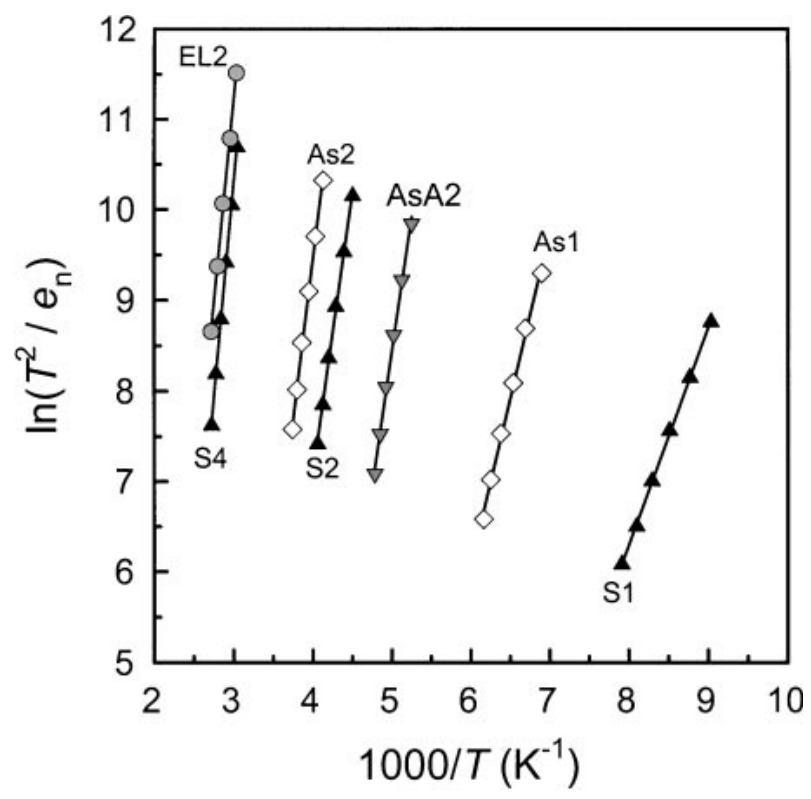

Fig. 4. Arrhenius plots from which the activation energies, $E_{\mathrm{t}}$, and apparent capture cross-sections, $\sigma_{\mathrm{a}}$, of the defects were extracted.

Table II. Electrical signatures of electron traps in as-grown and rapid thermally annealed $\mathrm{SiO}_{2}$-capped and $40 \mathrm{keV}$ As-implanted n-type GaAs epitaxial layers.

\begin{tabular}{|c|c|c|c|}
\hline Defect & $\begin{array}{c}\text { Activation energy, } \\
E_{\mathrm{t}}(\mathrm{eV})\end{array}$ & $\begin{array}{c}\text { Capture cross } \\
\text { section, } \sigma_{\mathrm{a}}\left(\mathrm{cm}^{2}\right)\end{array}$ & Remarks \\
\hline EL2 & $0.82 \pm 0.04$ & $2 \times 10^{-13}$ & as-grown \\
\hline $\mathrm{S} 1$ & $0.23 \pm 0.02$ & $7 \times 10^{-15}$ & $\begin{array}{c}E_{\mathrm{t}} \text { is field } \\
\text { dependent }{ }^{27,28)}\end{array}$ \\
\hline $\mathrm{S} 2$ & $0.53 \pm 0.02$ & $8 \times 10^{-14}$ & $\begin{array}{l}\text { superposition of } \\
2 \text { defects, including } \\
\mathrm{V}_{\mathrm{Ga}}-\mathrm{Si}_{\mathrm{Ga}}{ }^{27,28)}\end{array}$ \\
\hline $\mathrm{S} 4$ & $0.74 \pm 0.03$ & $2 \times 10^{-13}$ & $\begin{array}{l}\text { member of EL2 family } \\
\text { of defects }\end{array}$ \\
\hline As 1 & $0.32 \pm 0.02$ & $1 \times 10^{-15}$ & \\
\hline As2 & $0.61 \pm 0.03$ & $2 \times 10^{-14}$ & band of defects \\
\hline AsA2 & $0.49 \pm 0.02$ & $3 \times 10^{-14}$ & \\
\hline
\end{tabular}

$10^{13} \mathrm{~cm}^{-3}$. The Arrhenius plots from which the "signatures" (activation energy, $E_{\mathrm{t}}$, and apparent capture cross-section, $\sigma_{\mathrm{a}}$ ) of the defects were extracted are shown in Fig. 4. The electronic properties of the defects are summarized in Table II. Three prominent electron traps (S1, S2, and S4) were introduced in the impurity-free disordered layers. We have recently reported on the electronic and isochronal annealing properties of these defects. ${ }^{27,28)}$ Defect S2 was tentatively identified as a complex involving the gallium vacancy and $\mathrm{Si}$ dopant atoms (i.e. $\mathrm{V}_{\mathrm{Ga}}-\mathrm{Si}_{\mathrm{Ga}}$ ). ${ }^{27}$ ) This tentative identification was reached following the previous results of Kuzuhara et al. ${ }^{29)}$ and Ito et al. ${ }^{30)}$ Kuzuhara et al. studied the outdiffusion of $\mathrm{Ga}$ atoms from $\mathrm{GaAs}$ into $\mathrm{SiO}_{x} \mathrm{~N}_{y}$ capping layers following annealing at $850^{\circ} \mathrm{C}$ for $15 \mathrm{~min}$ in an $\mathrm{N}_{2}$ atmosphere. Furthermore, they used both horizontal Bridgeman and liquid encapsulated Czochralski grown ntype GaAs. That study found a correlation between the concentration of outdiffused $\mathrm{Ga}$ atoms and the concentration change of the electron trap EL5 $\left(E_{\mathrm{t}}=E_{\mathrm{C}}-0.45 \mathrm{eV}\right)$, and went on to propose that the likely candidate for EL5 would be a defect complex involving $\mathrm{V}_{\mathrm{Ga}}$, such as the Ga vacancyAs antisite complex, $\mathrm{V}_{\mathrm{Ga}}-\mathrm{As}_{\mathrm{Ga}}$, and $\mathrm{Ga}$ vacancy-Si donor complex, $\mathrm{V}_{\mathrm{Ga}}-\mathrm{Si}_{\mathrm{Ga}}{ }^{29)}$ It is worth pointing out here that Fig. 2 shows no significant free carrier compensation in the impurity-free disordered n-GaAs layer, which may at first appear to contradict the net donor loss in ref. 29. We believe that the minimal compensation in Fig. 2 relates to the much shorter annealing time of $30 \mathrm{~s}$ used in the present study compared to the longer ( $>40 \mathrm{~min}$ ) time used in ref. 29 to observe any donor loss. Furthermore, Ito et al. ${ }^{30)}$ reported on a similar defect in their $\mathrm{SiO}_{2}$-capped and annealed n-GaAs epilayers grown by molecular epitaxy on Si-substrates, and proposed that the defect, which they labelled R3, was a Sirelated defect. Hence, our previous identification of S2 as the $\mathrm{V}_{\mathrm{Ga}}-\mathrm{Si}_{\mathrm{Ga}}$ defect follows directly from our own results ${ }^{27)}$ and those reported in refs. 29 and 30. However, the filling pulsewidth dependence measurements (Fig. 5) clearly indicate that $\mathrm{S} 2$ is, in fact, the superposition of two defect peaks. The two distinct steps in the curve for S2 (open circles) is characteristic of two deep levels having different capturecross sections. It is pointed out here that the defect S2 reported in our previous investigations was measured using a pulse width, $t_{\mathrm{p}}=0.2 \mathrm{~ms},{ }^{27,28)}$ corresponding to the defect giving rise to the first plateau in Fig. 5. In the present study, we have used $t_{\mathrm{p}}=50 \mathrm{~ms}$ for all spectra shown in Fig. 3. The difference between the values of $E_{\mathrm{t}}$ reported here and in our previous studies can, therefore, be explained by the different filling pulse widths we have used in our studies. In our previous studies, ${ }^{27,28)}$ we have shown that defect S2 (measured using $t_{\mathrm{p}}=0.2 \mathrm{~ms}$ ) was introduced uniformly within the top $\sim 0.8 \mu \mathrm{m}$ of the impurity-free disordered GaAs samples (concentration $\sim 2 \times 10^{14} \mathrm{~cm}^{-3}$ for $900^{\circ} \mathrm{C}$ RTA), whereas the concentration of S4 decreased exponentially below the surface with a characteristic decay length, $\lambda \approx 0.2 \mu \mathrm{m} \quad$ (extrapolated surface concentration $\sim 3 \times$ $10^{14} \mathrm{~cm}^{-3}$ for $900^{\circ} \mathrm{C}$ RTA). We have previously proposed

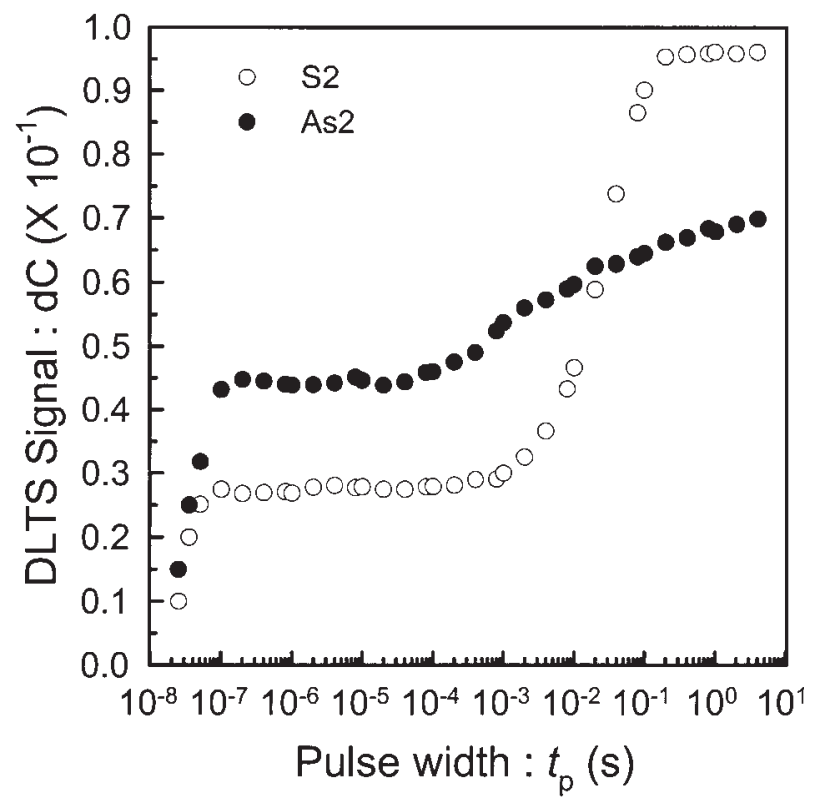

Fig. 5. Dependence of the peak intensities of defects S2 and As2 on the filling pulse width. The measurements were performed at the peak temperature of defects using a rate window of $(3.2 \mathrm{~s})^{-1}$. 
that S4 is a member of the EL2 family of defects, and those discussions can be found in refs. 27 and 28. The accurate depth profiling of $\mathrm{S} 1$, the origin of which is still unknown to us, is limited by its strong electric-field-assisted electron emission kinetics. $^{28)}$

The low-energy As ion implantation introduced two electron traps As1 and As2 in GaAs. For accurate determination of the "signature" of these defects, we have chosen reverse bias and filling pulse conditions so that we monitor only emission from defects in a region far enough below the surface where $\Delta N_{\mathrm{D}}<0.1$. Although, As2 exhibits the emission property (see Fig. 4) typical of a discrete electron trap, its broad and skewed profile [curve (c) in Fig. 3] suggests that it is most probably a superposition of several discrete levels or a continuous distribution of defect states. Indeed, the results shown in Fig. 5 (solid circles) reflect the non-discrete nature of As2. After RTA, one hole trap (AsA1) and one electron trap (AsA2) were observed in the heavily compensated ion implanted GaAs. We point out here that the severe carrier compensation incurred in the implanted samples prevented the quantitative defect depth profile measurements. However, the free carrier compensation observed from the doping profiles shown in Fig. 2, gives us a good indication of the relative concentration of defects and their diffusion lengths. Furthermore, we have not been able to determine the accurate "signature" of the hole trap AsA1. The leaky character of diodes fabricated on the implanted layers, as illustrated in Fig. 1 and the summary of results in Table I, prevented DLTS measurements above room temperature. Although the peak intensities of defects shown in Fig. 3 are of the same order magnitude, it does not follow that the concentrations of defects in the implanted and impurity-free disordered samples were the same, as evidenced by the vastly different extent of free carrier compensation (see Fig. 2) produced by the two methods.

The results presented above have shown that the impurityfree method retains the better electrical quality of the GaAs epilayers compared to ion implantation-induced disordering. During RTA, donor type defects may be introduced close to the $\mathrm{GaAs} / \mathrm{SiO}_{2}$ interface, due to either the outdiffusion of $\mathrm{Ga}$ atoms into the $\mathrm{SiO}_{2}$ or metallurgical reactions between the GaAs surface and the capping layer, which results in the decrease of barrier height of $\mathrm{Au}$ SBDs fabricated on the processed surface. This lowering in barrier height is accompanied by the degraded rectifying behavior of the diodes. However, the doping concentration beyond the zero bias depletion region (i.e. $\gtrsim 300 \mathrm{~nm}$ ) remains unchanged in comparison with the as-grown sample. The result shown in Fig. 2, therefore, demonstrate that the defect levels S1, S2, and S4 [see Fig. 3(a)] created in rapid thermally-annealed $\mathrm{SiO}_{2}$-capped n-GaAs epilayers are either inefficient electron trapping centers or were not introduced in high concentrations enough to produce similar reductions in free carriers as ion implantation. In contrast, free carrier compensation was observed in the low-energy As-implanted samples, with the effect being much more pronounced after RTA. The highly non-linear forward $I-V$ characteristic curves of diodes fabricated on the implanted layers revealed the current transport was most probably dominated by the recombination-generation mechanism. The high level of free carrier compensation in these samples was further evidence for the presence of high densities of electron trapping sites. Ionimplantation-induced and impurity-free disordering introduced different sets of defects in the n-type GaAs layers, which influenced charge transfer across the barrier differently. Furthermore, free carrier compensation up to depths exceeding $1.1 \mu \mathrm{m}$ demonstrates that the diffusion length of defects created by ion implantation could be very large.

Finally, we note that, although, the efficient free carrier trapping centers introduced by ion implantation may provide the better frequency response of optical devices as shown by previous studies, ${ }^{14,15)}$ their presence has detrimental influence on the electrical properties of the semiconductor as discussed above. The extent of free carrier compensation and leakage current that can be tolerated in optoelectronic devices have to be critically assessed before choosing the disordering method to be used.

\section{Conclusion}

In summary, the electrical characteristics of impurity-free disordered n-type GaAs are substantially different to the properties of samples implanted with $40 \mathrm{keV}$ As ions. I-V measurements revealed that Schottky barrier diodes fabricated on the $\mathrm{SiO}_{2}$-capped and annealed layers had better characteristics than diodes fabricated on the As-implanted samples. Compared to diodes fabricated on the as-grown samples, the decrease in the barrier height of Au SBDs fabricated on the impurity-free disordered layers could account for their poorer rectifying behavior. Furthermore, the highly non-linear forward characteristic curves on diodes deposited on the implanted surface showed that the recombination-generation mechanism dominated charge transfer across the barrier. The presence of recombination centers in the depletion region of ion implanted GaAs was further evidenced by the corresponding compensation in free carriers. The doping profiles also showed that the defects created in a region confined to the near-surface of implanted samples diffused to depths exceeding $\sim 0.8 \mu \mathrm{m}$. The enhanced diffusion of implantation-induced defects during RTA resulted in the further free carrier compensation deeper below the surface. The two methods used for creating damage to the GaAs lattice produced two different types of defects. Our results confirm that the defects created by the impurity-free method (S1, S2, and S4) were introduced in much lower concentrations than those created by ion implantation. Since DLTS measurements could not be performed above room temperature on the ion implanted samples, we have not been able to observe the $\operatorname{defect}(\mathrm{s})$ responsible for the large free carrier reduction in those samples. The results discussed in this report demonstrate unambiguously that the impurity-free method retains the good crystalline quality of the GaAs epilayers compared to implantation-induced disordering.

\section{Acknowledgements}

The Swedish Foundation for International Cooperation in Research and Higher Education (STINT) is kindly acknowledged for financial support. P. N. K. Deenapanray and H. H. Tan gratefully acknowledge the financial support of the Australian Research Council. 
1) For a comprehensive review of quantum well intermixing see, Semiconductor Quantum Wells Intermixing, ed. E. H. Li (Gordon and Breach, Amsterdam, 2000) Optoelectronic Properties of Semiconductors and Superlattices, Vol. 8.

2) J. H. Marsh: Semicond. Sci. Technol. 8 (1993) 1136.

3) R. M. Cohen, G. Li, C. Jagadish, P. T. Burke and M. Gal: Appl. Phys. Lett. 73 (1998) 803.

4) W. P. Gillin: Semiconductor Quantum Wells Intermixing, ed. E. H. Li (Gordon and Breach, Amsterdam, 2000) Optoelectronic Properties of Semiconductors and Superlattices, Vol. 8, p. 53, and references therein.

5) A. Pépin, C. Vieu, M. Schneider, H. Launois and Y. Nissim: J. Vac. Sci. Technol. B 15 (1997) 142.

6) R. M. Cohen: Mat. Sci. Eng. R20 (1997) 167.

7) P. N. K. Deenapanray and C. Jagadish: Electrochem. Solid-State Lett. 4 (2001) G11.

8) L. Fu, P. N. K. Deenapanray, H. H. Tan, C. Jagadish, L. V. Dao and M. Gal: Appl. Phys. Lett. 76 (2000) 837.

9) P. N. K. Deenapanray, H. H. Tan, L. Fu and C. Jagadish: Electrochem. Solid-State Lett. 3 (2000) 196.

10) A. Saher Helmy, S. K. Murad, A. C. Bryce, J. S. Aitchison, J. H. Marsh, S. E. Hicks and C. D. W. Wilkinson: Appl. Phys. Lett. 74 (1999) 732.

11) P. N. K. Deenapanray, H. H. Tan, M. I. Cohen, K. Gaff, M. Petravic and C. Jagadish: J. Electrochem. Soc. 147 (2000) 1950.

12) L. Fu, J. Wong-Leung J, P. N. K. Deenapanray, H. H. Tan, C. Jagadish, Bin Gong, R. N. Lamb, R. M. Cohen, W. Reichert, L. V. Dao and M. Gal: J. Appl. Phys. 92 (2002) 3579.

13) L. Fu, R. W. v. d. Heijden, H. H. Tan, C. Jagadish, L. V. Dao and M. Gal: Appl. Phys. Lett. 80 (2002) 1171.
14) L. V. Dao, M. B. Johnston, M. Gal, L. Fu, H. H. Tan and C. Jagadish: Appl. Phys. Lett. 73 (1998) 3408.

15) L. V. Dao, M. Gal, C. Carmody, H. H. Tan and C. Jagadish: J. Appl. Phys. 88 (2000) 5252.

16) S. M. Sze: Physics of Semiconductor Devices (Wiley Eastern, New Delhi, 1987) 2nd ed., pp. 35-38.

17) B. Elman, E. S. Koteles, P. Melman and C. A. Armiento: J. Appl. Phys. 66 (1989) 2104.

18) B. G. Svensson, K.-H. Rydén and B. M. S. Lewerentz: J. Appl. Phys. 66 (1989) 699

19) J. F. Ziegler, J. P. Biersack and U. Littmark: The Stopping and Range of Ions in Solids (Pergamon, New York, 1986) Vol. 1.

20) E. H. Rhoderick and R. H. Williams: Metal-Semiconductor Contacts (Clarendon Press, Oxford, 1988) pp. 89-140.

21) see ref. 16, p. 37.

22) see ref. 16, p. 286

23) Y. G. Wang and S. Ashok: J. Appl. Phys. 65 (1989) 2371.

24) P. N. K. Deenapanray, F. D. Auret and G. Myburg: J. Vac. Sci. Technol. B 16 (1998) 1873.

25) E. Grusell, S. Berg and L. P. Andersson: J. Electrochem. Soc. 127 (1980) 1573

26) L. C. Kimerling: J. Appl. Phys. 45 (1974) 1839.

27) P. N. K. Deenapanray, H. H. Tan, C. Jagadish and F. D. Auret: Appl. Phys. Lett. 77 (2000) 696.

28) P. N. K. Deenapanray, H. H. Tan, C. Jagadish and F. D. Auret: J. Appl. Phys. 88 (2000) 5255.

29) M. Kuzuhara, T. Nozaki and T. Kamejima: J. Appl. Phys. 66 (1989) 5833.

30) A. Ito, A. Kitagawa, Y. Tokuda, A. Usami, H. Kano, H. Noge and T. Wada: Semicond. Sci. Technol. 4 (1989) 416. 